\title{
$Q$-factor enhancement in all-dielectric anisotropic nanoresonators
}

\author{
Wei Liu, ${ }^{1,2, *}$ Andrey E. Miroshnichenko, ${ }^{2}$ and Yuri S. Kivshar ${ }^{2,3}$ \\ ${ }^{1}$ College of Optoelectronic Science and Engineering, National University of Defense Technology, Changsha, Hunan 410073, P. R. China \\ ${ }^{2}$ Nonlinear Physics Centre, Research School of Physics and Engineering, Australian National University, Canberra, ACT 0200, Australia \\ ${ }^{3}$ Department of Nanophotonics and Metamaterials, ITMO University, St. Petersburg 197101, Russia \\ (Received 5 July 2016; revised manuscript received 31 October 2016; published 23 November 2016)
}

\begin{abstract}
It is proposed and demonstrated that the $Q$ factor of optical resonators can be significantly enhanced by introducing an extra anisotropic cladding. We study the optical resonances of all-dielectric core-shell nanoresonators and show that radially anisotropic claddings can be employed to squeeze more energy into the core area, leading to stronger light confinement and thus significant $Q$-factor enhancement. We further demonstrate that the required homogenous claddings of unusual anisotropy parameters can be realized through all-dielectric multilayered isotropic structures. It is expected that the mechanism we have revealed not only offers extra flexibilities of resonance manipulations for conventional dielectric structures, but also may shed new light onto investigations into unconventional nanostructures consisting of two-dimensional materials that are intrinsically highly anisotropic.
\end{abstract}

DOI: 10.1103/PhysRevB.94.195436

\section{INTRODUCTION}

Optical resonators serve almost ubiquitously as an indispensable platform for efficient light-matter interactions and constitute the cornerstone of many fields not only in physics, but also in quite a few other interdisciplinary subjects in both chemistry and biology [1]. Different kinds of optical resonators correspond to different light-confining mechanisms, which can be roughly categorized as total internal reflection for whispering-gallery resonators [1-3], photonic band gap for photonic crystal cavities [4,5], Anderson localization for random resonators [6,7], electromagnetic surface waves for plasmonic and graphene resonators [8-14], etc. For opticalresonator-based fundamental research and applications, such as cavity-enhanced spectroscopy and sensing $[15,16]$, cavity optomechanics [17], nonlinear and quantum optics [18-20], high- $Q$ resonators are required to achieve a significant field confinement and low scattering loss rate, thus providing an efficient platform for strong light-matter interactions.

To support high- $Q$ resonances, the aforementioned mechanisms have different specific problems: photonic band gap and random resonators are limited by fabrication and cannot function beyond the diffraction limit; plasmonic and graphene resonators can be scaled down to the subwavelength spectral regime but, unfortunately, their performance is restricted by intrinsic losses of materials employed. Although an extremely-high- $Q$ whispering gallery resonator is almost free from intrinsic loss and can be easily fabricated, it relies on the excitation of the high-order modes (higher-order modes correspond to larger transverse light momentum, which leads to lower radiation losses and higher $Q$ factors $[2,13,21]$ ) and, thus, is inevitably compromised by the large footprint of the resonator and also the large mode volume. For some specific applications, such as lasing, high- $Q$ low-order modes are required, which nevertheless is very challenging to achieve in whispering-gallery-type resonators [22]. It was recently found that the principle of total internal reflection (TIR) can be extended to the interface of isotropic and anisotropic materials

*wei.liu.pku@gmail.com (or the so-called relaxed total internal reflection [23-25], RTIR) and it has been demonstrated that material anisotropy can be employed to accelerate the decay rate of evanescent waves, resulting in a stronger light-confinement capability in waveguiding systems [23,24].

In this paper, we study the resonances of all-dielectric core-shell cylindrical nanoresonators of nanowires from new perspectives of the total internal reflection at the interface of anisotropic media. To take advantage of the faster evanescent wave decay induced by the RTIR condition, we study a resonator that consists of an isotropic core and radially anisotropic cladding layer (the refractive index of the core is larger and smaller than the radial and transverse refractive index of the cladding layer, respectively). We show that such an anisotropic cladding can squeeze more energy of the mode into the core area, which results in stronger field confinement and thus larger $Q$ factors for resonances of various orders of the resonator. We further demonstrate that the cladding layer of naturally inaccessible anisotropy can be substituted by realistic multilayered isotropic metamaterial structures without compromising the property of $Q$-factor enhancement. The principle we have revealed is general, which can be applied to resonators of various shapes or of other sorts of anisotropy (such as magnetic anisotropy), to modes of higher orders, and to resonators operating at other spectral regimes. Such a mechanism cannot only play a critical role in the rapidly developing field of all-dielectric metamaterials $[25,26]$, but also inspires many other fundamental studies and applications based on two-dimensional (2D) materials that are intrinsically highly anisotropic [27,28], thus shedding light onto various subjects of cavity optomechanics, resonant quantum and nonlinear optics, thermal photonics, lasing and imaging, etc.

\section{SCATTERING CONFIGURATION AND THEORETICAL ANALYSIS}

We begin with one of the most fundamental structures of 2D nonmagnetic cylindrical resonators, as is shown in Fig. 1(a): the core layer (of radius $R_{1}$ ) is isotropic and the refractive index is $n_{1}$; the cladding layer (of radius $R_{2}$ ) is radially anisotropic 


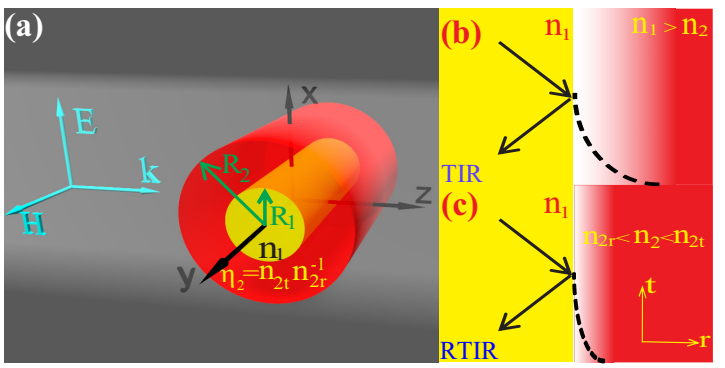

FIG. 1. (a) Schematic of the core-shell cylindrical resonator consisting of an isotropic core (refractive index $n_{1}$ and radius $R_{1}$ ) and a radially anisotropic cladding layer (radial index $n_{2 r}$, transverse index $n_{2 t}$, and radius $R_{2}$ ). The anisotropy parameter is defined as $\eta_{2}=n_{2 t} / n_{2 r}$ and the incident plane wave to excite the TM resonances is polarized along the $x$ direction. (b) Conventional TIR: the evanescent wave (dashed curve) resides within the lower-index $\left(n_{2}\right)$ isotropic medium and decays exponentially away from the boundary. (c) RTIR: the evanescent wave decays much faster away from the boundary, which is due to the anisotropy of the medium involved.

on the $x-z$ plane with radial index of $n_{2 r}$ and transverse (along the azimuthal direction) index of $n_{2 t}$; the anisotropy parameter is defined as $\eta_{2}=n_{2 t} / n_{2 r}$. The resonant modes of 2D cylindrical resonators can be classified into two sets: (i) the transverse-magnetic (TM) modes with no magnetic field in the $x-z$ plane, and (ii) the transverse-electric (TE) modes with no electric fields in the $x-z$ plane [29-31]. Since the TE resonances are not affected by the radial anisotropy of the shell layer, here we study only the TM resonances with electric fields in the $x-z$ plane. As a result, the incident plane wave is fixed to be polarized along the $x$ direction (in terms of the electric field). According to the proposed principle of RTIR [23,24], the radial anisotropy of the cladding layer can reduce the skin depth of the evanescent waves, as is shown schematically in Figs. 1(b) and 1(c): Fig. 1(b) shows the conventional TIR, where an evanescent wave (dashed curve) resides within the lower-index media, and it decays exponentially away from the boundary; Fig. 1(c) shows the RTIR geometry, where the evanescent waves decay faster, which is induced by the anisotropic layer employed.

As a first step, we study the scattering properties of the structure shown in Fig. 1(a). This problem can be solved analytically $[29,32,33]$, and the scattering efficiency (scattering cross section divided by the geometrical cross section of the structure $\pi R^{2}$ ) can be expressed as:

$$
\Gamma_{\mathrm{sca}}=\frac{2}{k R} \sum_{m=-\infty}^{\infty}\left|a_{m}\right|^{2},
$$

where $k$ is the angular wave number in the background material (vacuum in our work); $R$ is the radius of the outermost layer; $a_{0}$ and $a_{m}\left(a_{m}=a_{-m}\right)$ are the scattering coefficients, which depend on the anisotropy parameter (see the Appendix for more details). To be more specific, $a_{0}$ corresponds to the magnetic dipole (MD), which has all the electric fields along the transverse direction on the $x-z$ plane [30]. As the result, the MD resonance is only dependent on the transverse (azimuthal) refractive index of the cylindrical resonator, and consequently it is not affected by the radial anisotropy. In contrast, $a_{m}(m \neq 0)$ corresponds to the electric resonance of the $m$ th order [e.g., $a_{1}$ and $a_{2}$ correspond to the electric dipole (ED) and electric quadrupole (EQ), respectively], which is dependent on the anisotropy parameter.

\section{SCATTERING AND $Q$ FACTOR OF HOMOGENEOUS ISOTROPIC RESONATORS}

In Fig. 2(a) we show the scattering efficiency spectra for a homogenous and isotropic cylinder (radius $R=300 \mathrm{~nm}$ and refractive index $n=2$ ) in terms of the total scattering together with the contributions from the first three dominant resonances: $\mathrm{MD}, \mathrm{ED}$, and $\mathrm{EQ}$. Because $\mathrm{MD}$ is not affected by the radial anisotropy, throughout this work we focus only on the ED and EQ resonances which are both anisotropy sensitive. The indicated points D and Q in Fig. 2(a) correspond to the resonant positions of ED and EQ respectively: $\lambda_{D}=$ $1046 \mathrm{~nm}$ and $\lambda_{Q}=821 \mathrm{~nm}$. The corresponding near-field distributions $|\mathbf{E}|^{2}$ at those two points are shown in Figs. 2(b) and 2(c). We show here only the partial fields of the ED and EQ resonances, respectively (fields associated with other multipoles are neglected), which as a result exhibit typical pure $\mathrm{ED}$ and EQ characteristics, respectively.

Then, we study the $Q$ factors of the ED and EQ resonances of the homogeneous cylindrical nanowire. If we use $\omega_{\mathrm{m}}=$ $\omega_{\mathrm{m}}^{\prime}+i \omega_{\mathrm{m}}^{\prime \prime}\left(\omega_{\mathrm{m}}^{\prime \prime}>0\right)$ to denote the complex resonant angularfrequency of the $m$ th-order resonance, which corresponds to the singular point of the scattering matrix of the resonator, then
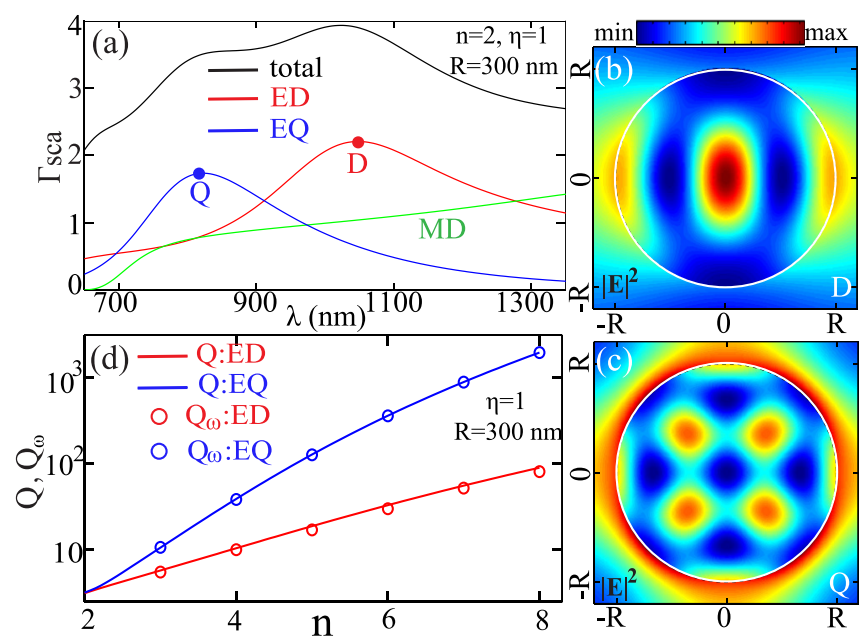

FIG. 2. (a) Scattering efficiency spectra for a homogeneous $(\eta=1)$ and isotropic cylindrical resonator $(n=2$ and $R=300 \mathrm{~nm})$. Both the total scattering efficiency spectrum (black curve) and those contributions from ED (red curve), MD (green curve), and EQ (blue curve) are shown. Two points $\mathrm{D}\left(\lambda_{D}=1046 \mathrm{~nm}\right)$ and $\mathrm{Q}$ $\left(\lambda_{Q}=821 \mathrm{~nm}\right)$, which correspond to the resonant positions of ED and EQ respectively, are marked in panel (a) and the corresponding near fields (partial electric-field intensity $|\mathbf{E}|^{2}$ ) are shown in panels (b) and (c). (d) The refractive-index dependence of the $Q$ factors for the ED and EQ supported. The curves correspond to the $Q$ factors obtained through Eq. (2) while circles correspond to $Q$ factors calculated based on FWHM of the scattering spectra. 
the corresponding $Q$ factor can be expressed as [3,29]:

$$
Q_{\mathrm{m}}=\frac{\omega_{\mathrm{m}}^{\prime}}{2 \omega_{\mathrm{m}}^{\prime \prime}} .
$$

It is worth noticing that, for a homogenous cylindrical resonator, the scattering matrix is dependent only on the normalized radius $\rho=k R$ and refractive index $n$. That is to say, for fixed mode order $m, \omega_{\mathrm{m}}$ is proportional to the resonator radius. As a result, according to Eq. (2), $Q_{\mathrm{m}}$ is $R$ independent and depends only on the refractive index $n$. The dependence of the $Q$ factors of ED and EQ on refractive index $n$ is shown in Fig. 2(d). As expected, with increasing $n$, the $Q$ factors of both ED and EQ increases monotonically, since larger momentum mismatch between the resonator and the background leads to stronger energy-confinement capability [3,13,29,34]. We note that, besides the definition shown in Eq. (2) [the results are shown by curves in Fig. 2(d)], $Q$ factors can be also obtained by calculating the full width at half maximum (FWHM) of the scattering spectrum [3]: $\mathrm{Q}_{\omega}=\omega_{0} / \Delta \omega_{1 / 2}$, where $\omega_{0}$ denotes the central resonant scattering frequency [such as the points indicated in Fig. 2(a)] and $\omega_{1 / 2}$ is width of the scattering spectrum curve measured between the points where the magnitudes are half of the maximum amplitude at the central resonant position. The results calculated through the scattering spectrum are also shown in Fig. 2(d) by circles and it is obvious they agree well with those obtained through Eq. (2). Throughout this paper, we focus on the ED and EQ resonances of the lowest frequency [besides the resonant peaks shown in Fig. 2(a), there are other scattering peaks for ED and EQ at higher frequencies $[29,35]]$ and calculate the $Q$ factor through Eq. (2). Nevertheless, it should be reminded that, for $\mathrm{ED}$ and $\mathrm{EQ}$ of higher frequencies and for other ways to define and/or calculate the $Q$ factors (or non-Lorentzian asymmetric linewidths of the scattering spectrum [36,37]), the conclusions drawn in our work are still valid.

\section{SCATTERING AND $Q$ FACTOR OF CORE-SHELL ANISOTROPIC RESONATORS}

As a next step, we switch to the cylindrical resonator with an anisotropic cladding and demonstrate how the RTIR mechanism can be employed to enhance the $Q$ factor and energy-confinement ability of the resonances. First we study the core-shell resonator with an isotropic core $\left(R_{1}=200 \mathrm{~nm}\right)$ and an anisotropic cladding. For better comparison with the homogenous resonator investigated above, we set the core index as $n_{1}=2$, cladding layer radius as $R_{2}=300 \mathrm{~nm}$, and the indexes of the cladding satisfy $n_{2 r} n_{2 t}=n_{1}^{2}$. As a result, the anisotropy parameter can be expressed as $\eta_{2}=\left(n_{2 t} / n_{1}\right)^{2}$. The scattering spectra with $\eta_{2}=\left(n_{2 t} / n_{1}\right)^{2}=4$ is shown in Fig. 3(a), where only the contributions from ED and EQ are shown [for a clearer comparison, the results of the homogenous case with the same outermost layer radius $R=300 \mathrm{~nm}$ shown already in Fig. 2(a) are replotted here by dashed curves]. As expected from the RTIR principle, the anisotropic cladding improves the energy confinement and thus can enhance the $Q$ factor for both ED and EQ, which can be justified by the presence of sharper and narrower scattering curves with anisotropy materials incorporated [3,29]. To quantitatively characterize the $Q$-factor enhancement, in Fig. 3(b) we show the dependence of $Q$ factors of both resonances on the anisotropy parameter, which further verifies the anisotropyinduced $Q$-factor enhancement. In principle, higher $Q$ factor indicates better energy confinement capability of the resonator. To confirm this directly, in Fig. 3(a), the positions of the ED and EQ resonances for both isotropic and anisotropic cases are indicated by $D, D^{\prime}$ and $Q, Q^{\prime}\left(\lambda_{D}=1046 \mathrm{~nm}\right.$, $\left.\lambda_{D^{\prime}}=1213 \mathrm{~nm}, \lambda_{Q}=821 \mathrm{~nm}, \lambda_{Q^{\prime}}=734 \mathrm{~nm}\right)$ and the corresponding total electric field (combining the contributions of all multipoles) intensity $\left(|\mathbf{E}|^{2}=\left|\eta^{-2} \mathbf{E}_{\mathrm{r}}+\mathbf{E}_{\mathrm{t}}\right|^{2}\right)$ are shown in Figs. 3(c)-3(f). Compared to the isotropic case [Figs. 3(c) and 3(e)], when anisotropy is introduced [Figs. 3(d) and 3(f)] more energy of the mode is squeezed into the core layer, indicating better energy confinement and as a result leading to more significant field enhancement [we note that, to make the field distributions clearer, different scales of the color bars for the fields at different points are employed in Figs. 3(c)-3(f)]. It is worth mentioning that, compared with the symmetric field distributions shown in Fig. 2, those shown in Fig. 3 are asymmetric because here we show the total fields contributed by all the multipoles. This is also the case for Figs. 4 and 5 .

To further quantify the capability of better energy confinement and more significant field enhancement, we denote the energy stored inside the core and shell cladding as $U_{c}$ and $U_{s}$, which can be defined respectively as

$$
\begin{aligned}
U_{\mathrm{c}} & =\iint_{\text {core }} n_{1}^{2}|\mathbf{E}(\mathbf{r})|^{2} d^{2} \mathbf{r}, \\
U_{\mathrm{s}} & =\iint_{\text {shell }}\left[n_{2 r}^{2}\left|E_{r}(\mathbf{r})\right|^{2}+n_{2 t}^{2}\left|E_{t}(\mathbf{r})\right|^{2}\right] d^{2} \mathbf{r},
\end{aligned}
$$

where $E_{r}(\mathbf{r})$ and $E_{t}(\mathbf{r})$ correspond to electric fields along the radial and transverse direction respectively, which can be calculated analytically $[29,32,38]$; the total stored energy inside the resonator is $U_{\mathrm{t}}=U_{\mathrm{c}}+U_{\mathrm{s}}$; and the core energy ratio is $U_{\mathrm{c}} / U_{\mathrm{t}}$. In Fig. $3(\mathrm{~g})$ we show the dependence of $U_{\mathrm{t}}$ and $U_{\mathrm{c}} / U_{\mathrm{t}}$ on the anisotropy parameter $\eta_{2}$ for the ED resonance. As is clearly shown, with larger $\eta_{2}$ both the total stored energy and the core energy ratio would increase, which proves convincingly tighter energy confinement and larger field enhancement inside the resonator that accompany the efficient $Q$-factor enhancement shown in Figs. 3(a) and $3(\mathrm{~b})$.

In the discussions above, we fixed the overall resonator radius to investigate the $Q$-factor dependence on anisotropy parameter. Now we fix the anisotropy parameter $\eta_{2}=4$ (with $R_{1}=200 \mathrm{~nm}, n_{1}=2$, and $n_{2 t}=4$ ) and study the relationship between $Q$ factor and the shell-cladding radius $R_{2}$. The results are summarized in Fig. 4(a) as solid curves for both ED and EQ resonances. It is clear that the $Q$ factor does not increase monotonically with increasing cladding radius, and there is actually an optimal radius of the shell to achieve the highest $Q$ factor for each resonance. To reveal the mechanism behind, we select three points for both $\operatorname{ED}\left[R_{2}(\mathrm{i})=250 \mathrm{~nm}\right.$, $R_{2}(\mathrm{ii})=330 \mathrm{~nm}$ (optimal radius for EQ), $\left.R_{2}(\mathrm{iii})=600 \mathrm{~nm}\right]$ and EQ $\left[R_{2}(\mathrm{iv})=250 \mathrm{~nm}, R_{2}(\mathrm{v})=300 \mathrm{~nm}\right.$ (optimal radius for $\mathrm{ED}), R_{2}(\mathrm{vi})=600 \mathrm{~nm}$ ] and show the corresponding total nearfield distributions $\left(|\mathbf{E}|^{2}\right)$ in Figs. 4(b)-4(g). It is shown that, 


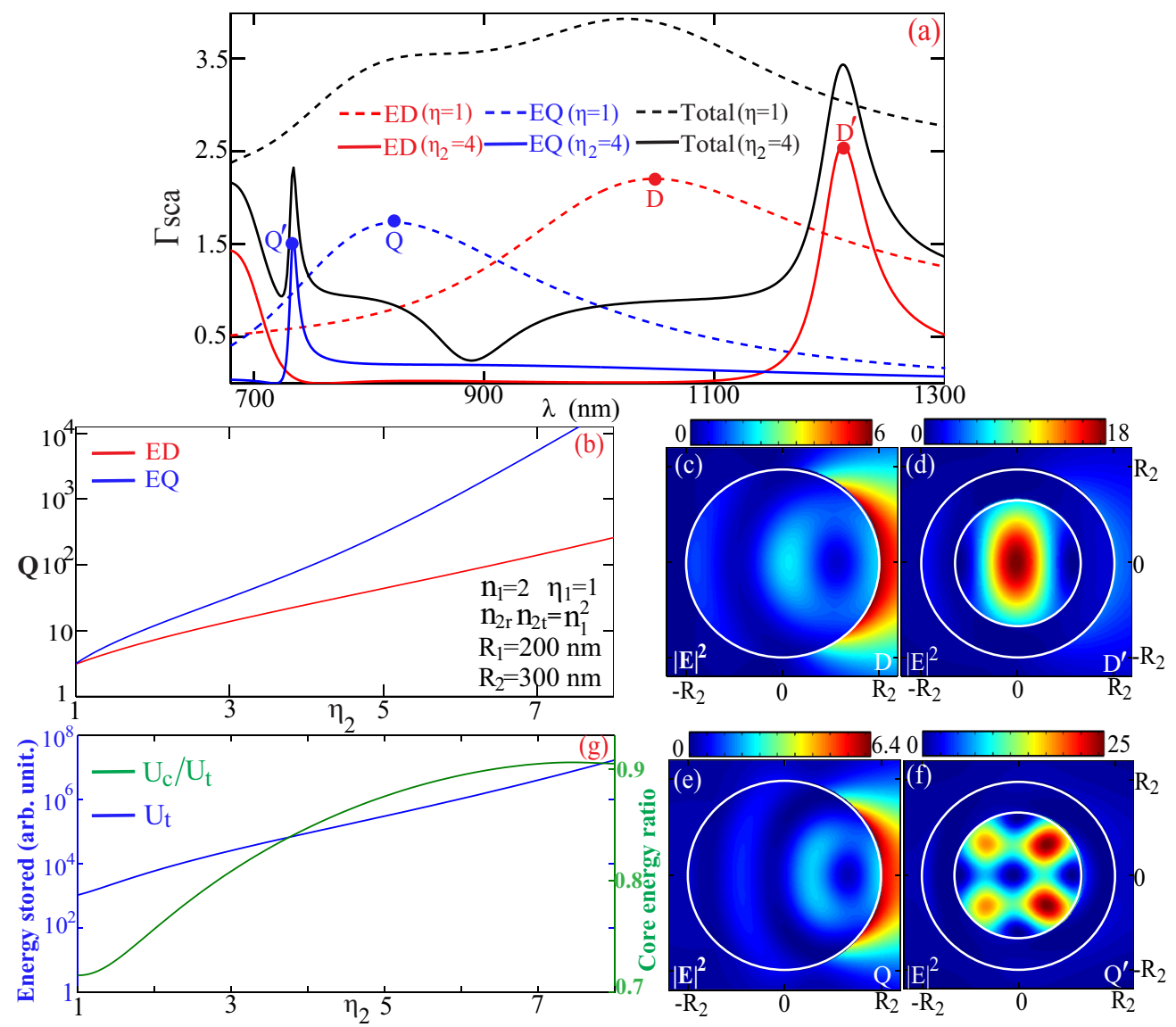

FIG. 3. (a) Scattering efficiency spectra for both ED (red curve) and EQ (blue curve) supported by the isotropic core-anisotropic shell cylindrical resonator of inner radius $R_{1}=200 \mathrm{~nm}\left(n_{1}=2\right)$ and outer radius $R_{2}=300 \mathrm{~nm}\left(\eta_{2}=4, n_{2 t}=4\right)$. For comparison, the ED and EQ scattering efficiency spectra for the homogenous and isotropic $(n=2)$ resonator of radius $300 \mathrm{~nm}$ are also shown as dashed curves. The total scattering efficiency spectrum is shown as black curves (dashed for the isotropic case and solid for the anisotropic case). Four resonant points for both cases $D, D^{\prime}$ and $Q, Q^{\prime}\left(\lambda_{D}=1046 \mathrm{~nm}, \lambda_{D^{\prime}}=1213 \mathrm{~nm}, \lambda_{Q}=821 \mathrm{~nm}, \lambda_{Q^{\prime}}=734 \mathrm{~nm}\right)$ are indicated and the corresponding total near-field distributions $\left(|\mathbf{E}|^{2}\right)$ are shown in panels (c)-(f). (b) The dependence of the $Q$ factor for both ED and EQ on anisotropy parameter. The indexes of the cladding layer is constrained by $n_{2 r} n_{2 t}=n_{1}^{2}=4$ and thus the anisotropy parameter is $\eta_{2}=\left(n_{2 t} / n_{1}\right)^{2}$. (g) The dependence of $U_{t}$ and $U_{c} / U_{t}$ for ED on the anisotropy parameter.

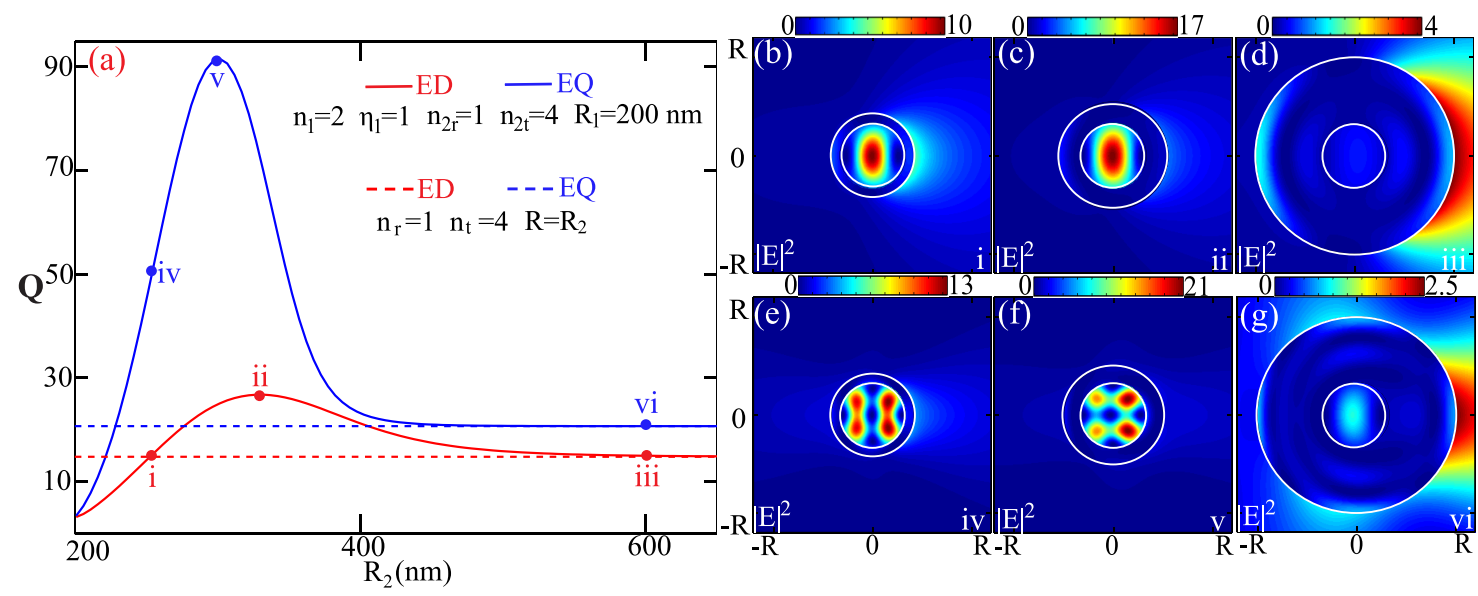

FIG. 4. (a) Dependence of $Q$ factor (solid red curve for ED and solid blue curve for EQ) on the shell-cladding radius $R_{2}$ with the following fixed parameters: $R_{1}=200 \mathrm{~nm}, n_{1}=2, \eta_{2}=4$, and $n_{2 t}=4$. The dashed curves correspond to the $Q$ factor (which is radius independent) for both ED (red) and EQ (blue) of the homogeneously anisotropic cylindrical resonator of $\eta=4$ and $n_{t}=4$. Three points have been indicated for the core-shell configuration [ED resonance: $R_{2}(\mathrm{i})=250 \mathrm{~nm}, R_{2}(\mathrm{ii})=330 \mathrm{~nm}, R_{2}(\mathrm{iii})=600 \mathrm{~nm}$; EQ resonance: $R_{2}(\mathrm{iv})=250 \mathrm{~nm}$, $\left.R_{2}(\mathrm{v})=300 \mathrm{~nm}, R_{2}(\mathrm{vi})=600 \mathrm{~nm}\right]$ and the total near-field distributions in terms of electric-field intensity $|\mathbf{E}|^{2}$ at those points are shown in panels (b) $-(\mathrm{g})$ where $R=600 \mathrm{~nm}$. 
before the optimal radius, for both resonances more energy is squeezed into the core region with increasing cladding radius, which leads to both $Q$-factor and near-field enhancement. In sharp contrast, after the optimal radius, with increasing $R_{2}$, more and more energy will leak out from the core, reducing both $Q$ factor and the field intensity within the resonator. This is due to the fact that, when the cladding layer is becoming sufficiently large, resonances can be formed within the cladding layer, with comparably negligible energy distributed within the core layer [see Figs. 4(d) and 4(g)]. To confirm directly this process of mode-distribution transformation between the core and the shell cladding, we plot the $Q$-factor-radius relationship of a homogeneous anisotropic cylindrical resonator with $\eta=4$ and $n_{2 t}=4$ for both ED (dashed red curve) and EQ (dashed blue curve) in Fig. 4(a): it is obvious that, for sufficiently large $R_{2}$, the $Q$ factor would converge to that of a homogeneous anisotropic cylinder (it is worth mentioning that, similar to the isotropic case studied in Fig. 2, the $Q$ factor of an anisotropic homogeneous resonator is also independent of the radius). The results presented here could be confusing at first glance, as it is taken for granted that increasing the anisotropic layer width would not break the condition of RTIR and thus would not result in a reduced $Q$ factor or near-field intensity. But one has to keep in mind that RTIR (and TIR itself) is a concept of geometric optics, and thus cannot be applied in a universal way. When the cladding layer is sufficiently large, potential distributions for photons would be changed drastically [21], resulting in more energy confinement within the cladding layer and undermining the features of the $Q$ factor and near-field enhancement.

\section{REALIZATION OF RELATIVELY LARGE EFFECTIVE RADIAL ANISOTROPY BASED ON MULTILAYERED STRUCTURES}

Up to now, we have demonstrated the efficient $Q$-factor enhancement based on anisotropic materials. Unfortunately, for natural materials the anisotropy parameters employed here may not be realistic. Such a problem is not insurmountable considering the recent development in the field of artificial metamaterials, where other extreme refractive indices and anisotropy parameters can be achieved [34,38-44]. Here, we employ a cladding layer consisting of multilayers of two kinds of realistic isotropic materials $\left(n_{l}\right.$ and $n_{h}$, respectively and $n_{l}<n_{h}$ ) to substitute the homogeneous anisotropic layer, as shown in Fig. 5(a). According to the effective-medium theory $[38,43,45]$, the effective indexes for the multilayered cladding on the $x-z$ plane are

$$
\begin{aligned}
& n_{2 \mathrm{r}}=n_{l} n_{h} / \sqrt{(1-f) n_{l}^{2}+f n_{h}^{2}}, \\
& n_{2 \mathrm{t}}=\sqrt{f n_{l}^{2}+(1-f) n_{h}^{2}},
\end{aligned}
$$

where $f$ (the thickness of all the layers of index $n_{l}$ divided by the whole thickness of the anisotropic cladding layer) is the filling factor of the layer of index $n_{l}$. When $f=0.5$ the largest anisotropy parameter can be achieved: $\eta_{2}=\left(n_{l}^{2}+n_{h}^{2}\right) / 2 n_{l} n_{h}$ for positive $n_{l}$ and $n_{h}$, and $n_{2 r} n_{2 t}=n_{l} n_{h}$. For a proof-ofconcept demonstration, we set $n_{l}=1$ and $n_{h}=4$, which leads to the following parameters for $f=0.5: n_{2 \mathrm{r}}=1.37$, $n_{2 \mathrm{t}}=2.92$, and $\eta_{2}=2.13$. The structure we employ is shown schematically in Fig. 5(a), where the cladding is made of

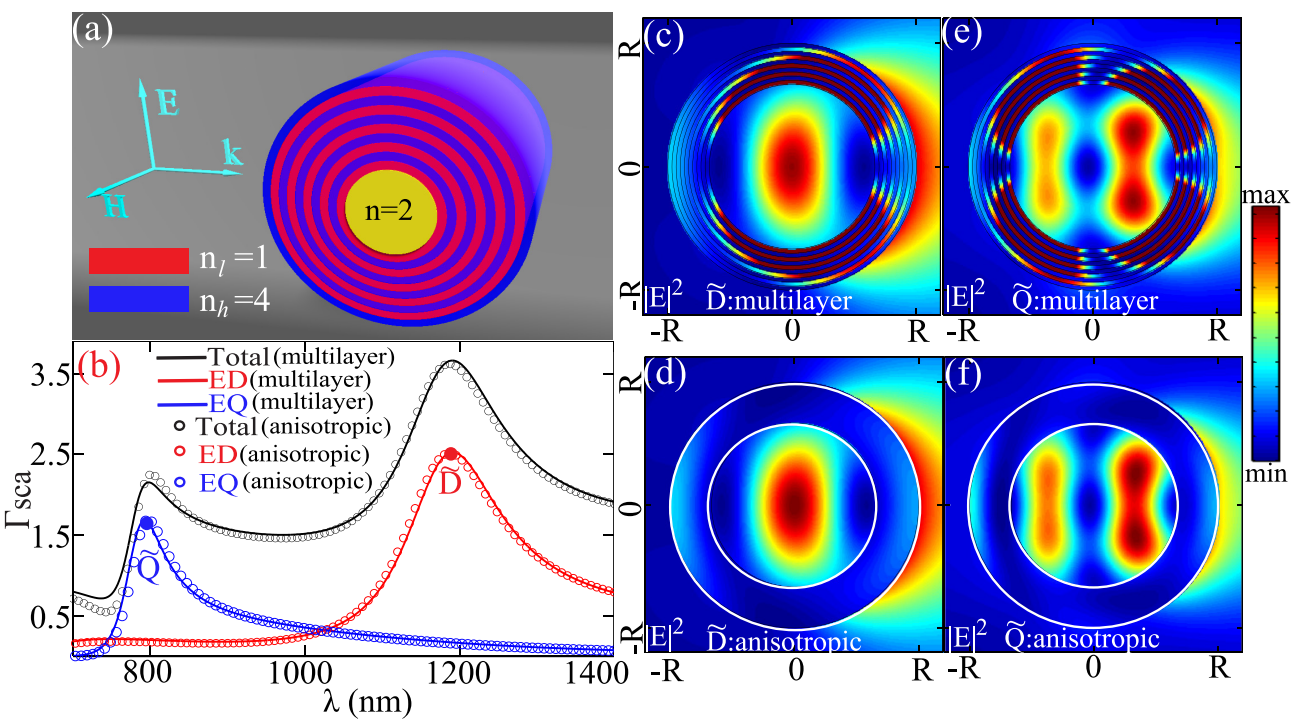

FIG. 5. (a) Schematic of the cylindrical resonator consisting of an isotropic core (refractive index $n_{1}=2$ and radius $R_{1}=200 \mathrm{~nm}$ ) and a multilayered cladding made of alternate $n=1$ and $n=4$ isotropic layers, and the width of both layers is $10 \mathrm{~nm}$. There are five layers of each medium, which leads to $f=0.5$ and $R_{2}=300 \mathrm{~nm}$. (b) Scattering efficiency spectra of the cylindrical resonator shown in panel (a) for both ED (red solid curves) and EQ (blue solid curves). The circles correspond to the scattering efficiency spectra of a two-layered cylindrical resonator with the same core as that shown in panel (a) and a homogenous anisotropic cladding layer of $n_{2 \mathrm{r}}=1.37, n_{2 \mathrm{t}}=2.92$, and $R_{2}=300 \mathrm{~nm}$ : both the spectra of ED (red circles) and EQ (blue circles) are shown. The total scattering efficiency spectrum is also shown by black curve (multilayer) and black circles (anisotropic cladding). Two points $\tilde{D}\left(\lambda_{\tilde{D}}=1191 \mathrm{~nm}\right)$ and $\tilde{Q}\left(\lambda_{\tilde{Q}}=791 \mathrm{~nm}\right)$, which correspond to the resonant positions of ED and EQ, respectively, are marked in panel (b) and the corresponding total near-field distributions $\left(|\mathbf{E}|^{2}\right)$ are shown in panels (c) and (e) for the multilayered isotropic cylindrical resonator, and in panels (d) and (f) for the two-layered core-shell resonator with a homogenous anisotropic cladding. 
alternate $n_{l}$ and $n_{h}$ layers and the width of both layers is $10 \mathrm{~nm}$ [which is far smaller then the effective wavelength and then the effective-medium theory Eq. (4) can be applied]. There are five layers of each medium, which makes $f=0.5$ and the overall cladding layer of radius $R_{2}=300 \mathrm{~nm}$ (for direct comparisons with the previous discussions, the core is set to be isotropic with radius $R_{1}=200 \mathrm{~nm}$ and of $n_{1}=2$ ). The scattering efficiency spectra of such an isotropic 11-layered cylindrical resonator can be calculated analytically [29] and the results are shown in Fig. 5(b) by solid curves for both ED and EQ resonances. It is worth mentioning that the specific layer width or the periodicity or the layer number is not important (actually they all can be random) as long as each layer width is far smaller than the wavelength when the effective-medium theory can be applied. This distinguishes our design from those based on photonic band-gap structures [4,5]. To verify the effective medium theory applied for the multilayered cladding, we also show in Fig. 5(b) by circles the scattering efficiency spectra when the multilayered cladding is replaced by a homogenous anisotropic layer [according to Eq. (4)] of $n_{2 \mathrm{r}}=1.37, n_{2 \mathrm{t}}=2.92$, and $n_{2 r} n_{2 t}=n_{1}^{2}=4$. It is obvious that both sets of results agree excellently well, indicating the same $Q$ factor for both cases [this has already been included in Fig. 3(b)]. The two indicated points $\tilde{D}$ and $\tilde{Q}$ in Fig. 5(a) correspond to the resonant position of ED and EQ, respectively: $\lambda_{\tilde{D}}=1191 \mathrm{~nm}$ and $\lambda_{\tilde{Q}}=791 \mathrm{~nm}$. The corresponding total near-field distributions $\left(|\mathbf{E}|^{2}\right)$ are shown in Figs. 5(c) and 5(e) for the multilayered isotropic cylindrical resonator, and in Figs. 5(d) and 5(f) for the core-shell anisotropic resonator. The strong-field confinement is clearly demonstrated by comparing Figs. 5(c)-5(f) with Figs. 3(c) and 3(e). Although the field distributions within the cladding layer are contrastingly different (the fields are significantly enhanced within the $n=1$ layers due to the continuity condition along the radial direction, which is similar to that shown in Ref. [46]), the fields inside the core and outside the resonator are almost the same, proving further the feasibility of applying the RTIR principle to obtain high- $Q$ cavity resonators.

It is worth mentioning that, here for the proof-of-concept demonstration, we set all-dielectric materials to relatively low index contrast $\left(n_{l}=1\right.$ and $\left.n_{h}=4\right)$, which results in a moderately effective anisotropy parameter $\left(\eta_{2}=2.13\right)$ and a not-so-significant $Q$-factor enhancement [see Fig. 3(b)]. Nevertheless, we should keep in mind that the mechanism we have revealed is scalable and applicable at other spectral (such as microwave and terahertz) regimes, where much higher anisotropy parameter (index contrast) and thus more significant $Q$-factor enhancement can be obtained. Moreover, many other extremely unusual anisotropy parameters (including negative and complex ones) can be made available for realistic applications relying on the artificial metamaterials or the recently emerging various sorts of $2 \mathrm{D}$ materials that are intrinsically highly anisotropic [34,38-44]. Although intrinsic losses are usually inevitably present in such artificial metamaterials, which can comprise the effect of $Q$-factor enhancement and energy confinement. Basically there would be a trade-off between the effects of loss and anisotropy, where there must be a specific region where the $Q$ factor can be mostly significantly enhanced. The competition between loss and anisotropy with regard to the $Q$-factor enhancement is out of the scope of this paper, but it is expected that, relying on the artificial metamaterials and/or 2D materials, we would be able the design proper structures to balance the effects of loss and anisotropy to obtain the optimum $Q$-factor enhancement.

\section{CONCLUSIONS AND OUTLOOK}

In summary, based on the recently proposed principle of light localization in anisotropic media, we studied alldielectric cylindrical resonators with an isotropic core and anisotropic cladding, focusing on the $Q$-factor enhancement of the resonances supported. Due to the RTIR effect, we demonstrate that more energy can be confined within the core-layer structure, which leads to simultaneous $Q$-factor and near-field enhancement for the resonances. We also show that, with a fixed radial anisotropy parameter of the cladding, there exist optimal cladding layer widths to achieve the highest $Q$ factors for resonances of different orders, thus succeeding in revealing the limitation of the application of RTIR principle to resonators. To verify the feasibility of our approach to achieve high- $Q$ resonators, we have shown that naturally inaccessible anisotropy parameters of the cladding can be realized by employing multilayered isotropic metamaterial structures with the property of significant $Q$-factor enhancement preserved.

It is worth mentioning that, although in this work we confine our study to the two lowest order of modes (dipole and quadrupole modes) of cylindrical structures, the principle we have revealed is quite general. The $Q$-factor enhancement can certainly be achieved in spherical resonators and resonators of other shapes, for other kinds of anisotropy such as magnetic anisotropy and for modes of higher orders. Actually, the higher the mode order is, the stronger the $Q$-factor enhancement effect would be [see Fig. 3(b)]. Since more energy is confined within the core of the resonator by the RTIR effect, it is expected that, for a cluster of such resonators, the near-field coupling between them can significantly be reduced $[47,48]$. Here in this work, we constrain the indexes of the cladding layer by $n_{2 r} n_{2 t}=n_{1}^{2}$, which is implemented only for a direct comparison with the homogeneous isotropic resonator of $n=n_{1}$. Basically there is no compulsory constraint for the indexes of the cladding layer, and beyond this imposed constraint with higher anisotropy parameters, for example when $n_{2 r}=1, n_{2 t}>n_{1}^{2}$, more significant $Q$-factor enhancement can be achieved. We believe that our work of employing the effective anisotropic media to enhance the $Q$ factor opens a new dimension of freedom for manipulations of various resonators, which can incubate many new resonator-based fundamental studies and applications in the fields of cavity-enhanced spectroscopy and sensing, cavity optomechanics, resonant quantum and nonlinear optics, lasing and imaging, etc.

\section{ACKNOWLEDGMENTS}

We acknowledge financial support from the National Natural Science Foundation of China (Grant No. 11404403), the Australian Research Council and the Basic Research Scheme of College of Optoelectronic Science and Engineering, National University of Defence Technology. W.L. thanks 
the Nonlinear Physics Centre for their warm hospitality during his visit to Canberra.

\section{APPENDIX}

The seminal problem of two-dimensional (2D) plane-wave scattering by cylindrical resonators (single or multilayered) consisting of isotropic materials has been studied analytically [29] [e.g., for the scattering configuration shown in Fig. 5(a) with the corresponding scattering efficiency shown in Fig. 5(b) by solid curves]. Such analytical investigations have also been extended to $2 \mathrm{D}$ cylindrical resonators made of radially anisotropic materials [32,33] [e.g., for the configuration shown in Fig. 1(a)]. It has been revealed that, for both isotropic and radially anisotropic cases, the expressions for the field-expansion coefficients (both inside and outside the resonator) and far-field scattering properties are identical [see Eq. (1)], except that, in the anisotropic case, the orders of some Bessel and Hankel functions need to be modified by the anisotropy parameter.
To be more specific, we take for example the simplest case of a homogeneous radially anisotropic cylinder (radius $R$ and anisotropy parameter $\eta$ ) in vacuum. The scattering efficiency can be expressed by Eq. (1) where the scattering coefficients for the incident plane waves of TM polarizations can be expressed as $[29,32]$

$$
a_{ \pm m}=\frac{n_{t} J_{\tilde{m}}\left(n_{t} k R\right) J_{m}^{\prime}(k R)-J_{m}(k R) J_{\tilde{m}}^{\prime}\left(n_{t} k R\right)}{n_{t} J_{\tilde{m}}\left(n_{t} k R\right) H_{m}^{\prime}(k R)-H_{m}(k R) J_{\tilde{m}}^{\prime}\left(n_{t} k R\right)},
$$

where $\tilde{m}$ is the modified function order $\tilde{m}=m \eta, J$ and $H$ are respectively the Bessel and Hankel functions of the first kind [29], and the accompanying primes indicate their differentiation with respect to the entire argument. Obviously the results reduce to the those of isotropic case when $\eta=1$. It is clear that the MD mode of $m=0$ is independent of the anisotropy, which is consistent with our former argument based on the field distribution. Similar to Eq. (A1), all the field expansion coefficients within the resonator can be obtained in all the layers by simply modifying the function order when the radially anisotropic materials are present. As a result, the energy stored within the resonator [see Eq. (3)] can also be calculated analytically.
[1] K. Vahala, Optical Microcavities (World Scientific, London, 2004).

[2] C. C. Lam, P. T. Leung, and K. Young, J. Opt. Soc. Am. B 9, 1585 (1992).

[3] A. Yariv and P. Yeh, Photonics: Optical Electronics in Modern Communications (Oxford University Press, Inc., 2006).

[4] Y. Akahane, T. Asano, B. S. Song, and S. Noda, Nature (London) 425, 944 (2003).

[5] J. D. Joannopoulos, Photonic Crystals: Molding the Flow of Light, 2nd ed. (Princeton University Press, Princeton, 2008).

[6] E. Abrahams, 50 Years of Anderson Localization (World Scientific, 2010), Vol. 24.

[7] M. Segev, Y. Silberberg, and D. N. Christodoulides, Nat. Photonics 7, 197 (2013).

[8] A. D. Boardman, Electromagnetic Surface Modes (Wiley, Chichester, New York, 1982).

[9] J. Polo, T. Mackay, and A. Lakhtakia, Electromagnetic Surface Waves: A Modern Perspective (Newnes, 2013).

[10] S. A. Maier, Plasmonics: Fundamentals and Applications (Springer, New York, 2007).

[11] B. Min, E. Ostby, V. Sorger, E. Ulin Avila, L. Yang, X. Zhang, and K. Vahala, Nature (London) 457, 455 (2009).

[12] F. H. Koppens, D. E. Chang, and F. J. Garcia de Abajo, Nano Lett. 11, 3370 (2011).

[13] W. Liu, R. F. Oulton, and Y. S. Kivshar, Sci. Rep. 5, 12148 (2015).

[14] R. Li, X. Lin, S. Lin, X. Liu, and H. Chen, Opt. Lett. 40, 1651 (2015).

[15] G. Gagliardi and H. P. Loock, Cavity-Enhanced Spectroscopy and Sensing (Springer, 2014).

[16] J. Zhu, S. K. Ozdemir, Y. F. Xiao, L. Li, L. He, D. R. Chen, and L. Yang, Nat. Photonics 4, 46 (2010).

[17] M. Aspelmeyer, T. J. Kippenberg, and F. Marquardt, Rev. Mod. Phys. 86, 1391 (2014).
[18] R. E. Slusher, L. W. Hollberg, B. Yurke, J. C. Mertz, and J. F. Valley, Phys. Rev. Lett. 55, 2409 (1985).

[19] S. Haroche and J. M. Raimond, Exploring the Quantum: Atoms, Cavities, and Photons (Oxford University Press, 2006).

[20] K. Srinivasan and O. Painter, Nature (London) 450, 862 (2007).

[21] B. R. Johnson, J. Opt. Soc. Am. A 10, 343 (1993).

[22] M. Naffouti, T. David, A. Benkouider, L. Favre, A. Ronda, I. Berbezier, S. Bidault, N. Bonod, and M. Abbarchi, Nanoscale 8, 2844 (2016).

[23] S. Jahani and Z. Jacob, Optica 1, 96 (2014).

[24] S. Jahani and Z. Jacob, J. Opt. Soc. Am. B 32, 1346 (2015).

[25] S. Jahani and Z. Jacob, Nat. Nanotechnol. 11, 23 (2016).

[26] A. I. Kuznetsov, A. E. Miroshnichenko, M. L. Brongersma, Y. S. Kivshar, and B. Luk'yanchuk, Science 354, 2472 (2016).

[27] F. Xia, H. Wang, D. Xiao, M. Dubey, and A. Ramasubramaniam, Nat. Photonics 8, 899 (2014).

[28] Z. Liu and K. Aydin, Nano Lett. 16, 3457 (2016).

[29] M. Kerker, The Scattering of Light, and other Electromagnetic Radiation (Academic Press, New York, 1969).

[30] W. Liu, A. E. Miroshnichenko, R. F. Oulton, D. N. Neshev, O. Hess, and Y. S. Kivshar, Opt. Lett. 38, 2621 (2013).

[31] W. Liu, J. Zhang, B. Lei, H. Hu, and A. E. Miroshnichenko, Opt. Lett. 40, 2293 (2015).

[32] H. L. Chen and L. Gao, Phys. Rev. A 86, 033825 (2012).

[33] H. Chen and L. Gao, Opt. Express 21, 23619 (2013).

[34] X. Yang, J. Yao, J. Rho, X. Yin, and X. Zhang, Nat. Photonics 6, 450 (2012).

[35] W. Liu, Opt. Express 23, 14734 (2015).

[36] Z. Ruan and S. Fan, J. Phys. Chem. C 114, 7324 (2009).

[37] M. I. Tribelsky and A. E. Miroshnichenko, Phys. Rev. A 93, 053837 (2016).

[38] K. H. Kim, Y. S. No, S. Chang, J. H. Choi, and H. G. Park, Sci. Rep. 5, 16027 (2015).

[39] C. M. Soukoulis and M. Wegener, Nat. Photonics 5, 523 (2011). 
[40] N. I. Zheludev and Y. S. Kivshar, Nat. Mater. 11, 917 (2012).

[41] M. Choi, S. H. Lee, Y. Kim, S. B. Kang, J. Shin, M. H. Kwak, K. Y. Kang, Y. H. Lee, N. Park, and B. Min, Nature (London) 470, 369 (2011).

[42] C. Wu, A. Salandrino, X. Ni, and X. Zhang, Phys. Rev. X 4, 021015 (2014).

[43] A. Poddubny, I. Iorsh, P. Belov, and Y. Kivshar, Nat. Photonics 7, 948 (2013).
[44] W. Liu, B. Lei, J. Shi, and H. Hu, Sci. Rep. 6, 34775 (2016).

[45] V. Agranovich and V. Kravtsov, Solid State Commun. 55, 85 (1985).

[46] R. F. Oulton, V. J. Sorger, D. A. Genov, D. F. P. Pile, and X. Zhang, Nat. Photonics 2, 496 (2008).

[47] W. Liu, A. E. Miroshnichenko, D. N. Neshev, and Y. S. Kivshar, ACS Nano 6, 5489 (2012).

[48] A. E. Miroshnichenko and Y. S. Kivshar, Nano Lett. 12, 6459 (2012). 